\title{
Cooling of neutron stars with quark-hadron continuity
}

\author{
Tsuneo Noda ${ }^{1, *}$, Nobutoshi Yasutake $^{2,}$, Masa-aki Hashimoto ${ }^{3,}$, Toshiki Maruyama ${ }^{4,}$, and \\ Toshitaka Tatsumi ${ }^{5}$, \\ ${ }^{1}$ Kurume Institute of Technology, 2228-66 Kamitsu-machi, Kurume, Fukuoka 830-0052, Japan \\ ${ }^{2}$ Chiba Institute of Technology, Chiba 275-0023, Japan \\ ${ }^{3}$ Department of Physics, Kyushu University, Fukuoka 819-0395, Japan \\ ${ }^{4}$ Advanced Science Research Centre, Japan Atomic Energy Agency, Ibaraki 319-1195, Japan \\ ${ }^{5}$ Osaka Sangyo University, Osaka 574-8530, Japan
}

\begin{abstract}
Neutron stars are high-density objects formed by the gravitational collapse of massive stars, and the whole star can be likened to a giant nucleus. The interior of a neutron star is considered to contain exotic particles and states which do not appear in a normal nucleus. The internal states are constrained by observations of masses and radii via the equation of state of highly dense nuclear matter. Within these constraints, a variety of exotic states have been discussed. The internal state of neutron stars is closely related to its neutrino emission process, which cools the star from the inside. This effect can be compared with observations of the surface temperature of neutron stars. However, despite the wide range of observations of neutron stars, the nature of the neutron star matter remains uncertain. We consider quark matter as an exotic state and perform cooling calculations for neutron stars, incorporating the effects of nucleon superfluidity and quark colour superconductivity. We take into account the "quark-hadron continuity", in which the neutron superfluidity is succeeded by the d quark pairing. Furthermore, we obtained the range of the neutron star cooling curve, taking into account the difference in surface temperature due to the composition of the surface layer. We found that the existence of quark matter causes strong neutrino emission from quarks, which is moderately suppressed by superfluidity and superconductivity, and can explain the cold surface temperature of neutron stars.
\end{abstract}

\section{Introduction}

Neutron stars are extremely dense objects, which can be likened to giant atomic nuclei. They are born at high temperature during supernova explosions, but have no internal heat source, and are cooled by neutrino and $\mathrm{X}$-ray radiation processes. The dominant cooling process in neutron star cooling is neutrino emission in the early stages, and X-ray emission from the surface in the later stages. Neutrino emission is strongly dependent on the state of the dense nuclear matter inside, and there is a strong relationship between the cooling of the neutron star and the state of the dense nuclear matter.

The state of dense nuclear matter constituting a neutron star can be exotic and different from that of normal nuclear matter. Such exotic states have a different neutrino emission

\footnotetext{
*e-mail: noda@kurume-it.ac.jp
} 
mechanism to that of normal matter and have a significant impact on the thermal evolution of neutron stars. Exotic states are explored in the terrestrial experiments, but the internal states of neutron stars are relatively cold compared to their density, and it is very difficult to reproduce them in heavy-ion collisions. The most effective method to explore the internal states of neutron stars is to compare theoretical calculations with observations.

The states of the dense nuclear matter in neutron stars must be common to all neutron stars, and therefore the equation of state (EoS) describing the state of matter must also be common. Recent observations of neutron star masses of $2 M_{\odot}$ have resulted in a very strong constraint on the EoS. The maximum mass determined by the EoS and the TOV equation must exceed $2 M_{\odot}$. Furthermore, the EoS corresponds to the state of internal matter and is directly linked to the neutrino emission process.

The simplest neutrino emission process is the $\beta$ decay of neutrons and their inverse reaction, known as the Direct URCA (DU), but these processes are forbidden in degenerate neutron stars with a low proton fraction $(<1 / 9)$ because they violate the momentum conservation. The Modified URCA (MU) process, which solves the momentum conservation problem by involving other nucleons, is thought to be the dominant neutrino emission process in neutron stars. However, the MU process has a much lower emissivity than the DU process and cannot explain the temperatures of some cold neutron stars. To account for the observed temperatures of neutron stars, we would need a strong neutrino emission process, such as the DU process, or an exotic state with similar strong cooling which are only valid for some stars. Such a state above the critical density would lead to weak neutrino emission from normal nuclear matter in lighter neutron stars, where the central density does not reach the critical density, and to strong neutrino emission in heavier stars. This way can explain the observed surface temperatures under a unified EoS.

We investigate the thermal evolution of neutron stars by constructing a model of the neutron star cooling, considering the EoS in which nucleons decay into quark matter at high densities. The model takes into account the effects of nucleon superfluidity and quark colour superconductivity (CSC), as well as the quark-hadron continuum in superfluidity. And we investigate the effects of these "superstates".

\section{Cooling Models}

We consider that the neutron stars consist the standard hadronic matter at low density, and deconfined quark matter at high density. The hadronic matter transits into the nucleon superfluidity state below the critical temperature, and quark matter transits into CSC state. The effects of superfluidity/superconductivity included to the neutrino emissivities. We take account of ${ }^{1} S_{0}$ and ${ }^{3} P_{2}$ states for neutrons and ${ }^{1} S_{0}$ state for protons in the hadronic phase. For quark phase, we include the effects of Colour-Flavour-Locking (CFL) paring, Two-FlavourSuperconductivity ( $2 \mathrm{SC}$ ) pairing or " $2 \mathrm{SC}+\mathrm{X}$ " phase.

The superfluid/superconducting phases have two effects on neutrino emissivities. One is that they cause strong neutrino emission during the transition from the normal phases[1], and the another one is that they suppress other neutrino emission process after the transition. The suppressed neutrino emissivities can be obtained with the gap energy $\Delta$ and the original emissivity $\varepsilon_{v}$ as $\varepsilon_{v} \exp \left(-\Delta / k_{\mathrm{B}} T\right)$, here $k_{B}$ denotes the Boltzmann constant and $T$ is the temperature of the layer. We construct the models of the critical temperature as parametrized functions which depend on the density as Figure 1 for nucleon superfluidity.In this calculation, four models were created by varying the maximum critical temperature and fixing the density at which the critical temperature is maximum. The models were labelled A to D in ascending order of the critical temperature. 

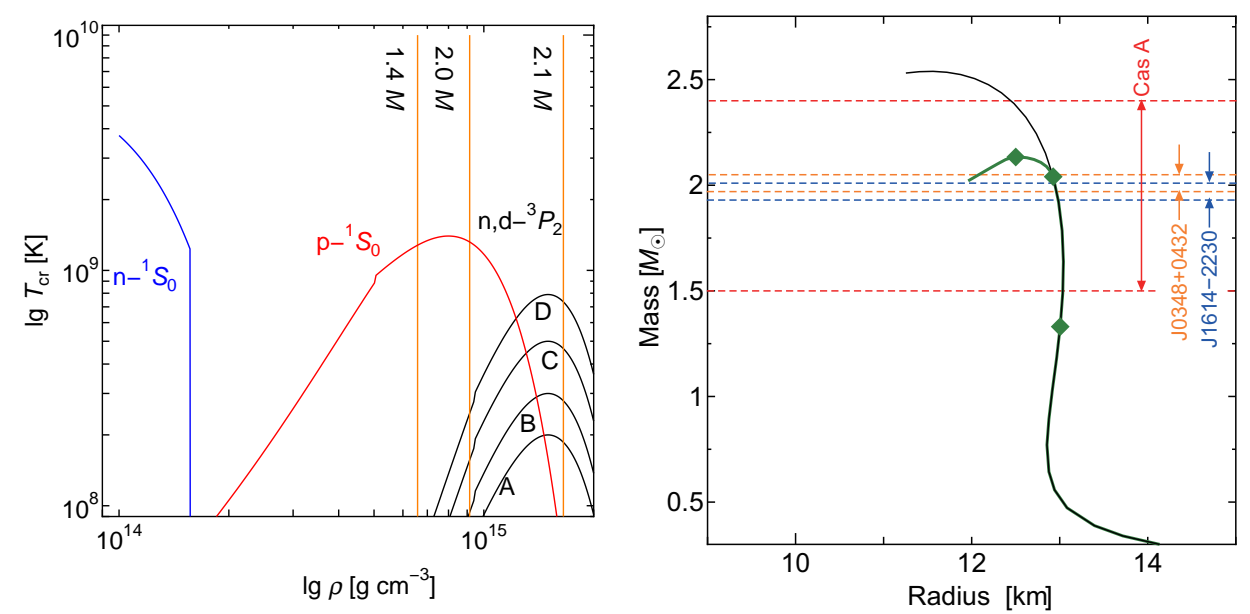

Figure 1. Superfluid temperatures of each state. Figure 2. $M-R$ relation of the adopted EoS. Black lines denote the difference of neutron ${ }^{3} P_{2}$ Green dots denotes the calculation models. model.

For the CSC, we assume the gap $\Delta$ is large enough comparing with the temperature $T$ (ie. $\Delta>10 \mathrm{MeV}$ ). All colours/flavours of quarks are in superconducting state in the CFL state, and 2 of 3 colours are in superconducting state in 2SC state. The CFL state strongly suppresses the neutrino emissivity of quarks, and the 2SC state also suppresses it in about $1 / 3$. We focus on the concept of "quark hadron continuity" of superfluidity. In this concept, the neutron ${ }^{3} P_{2}$ superfluidity is taken over by the $d$-quark ${ }^{3} P_{2}$ pairing during the crossover from hadronic matter to quark matter, and this $d$-quark paring can coexist with the 2SC CSC of other quarks[2]. We assume that the continuity occurs during the transition and it carries the superfluid effect on the neutrino emission. We also assume that the density dependence of the critical temperature of the $d$-quark ${ }^{3} P_{2}$ paring is connected with that of the neutron ${ }^{3} P_{2}$ superfluidity. We include the quark-hadron continuity effect to our calculation, as the name of " $2 \mathrm{SC}+\mathrm{X}$ " for the comparison with other CSC parings (2SC, CFL). 2SC+X state are combination of $2 \mathrm{SC}$ state and $d$-quark ${ }^{3} P_{2}$ state which is carried from the neutron ${ }^{3} P_{2}$ state. We treat the $2 \mathrm{SC}$ component as pure $2 \mathrm{SC}$ state, and the ${ }^{3} P_{2}$ component as the extension of neutron ${ }^{3} P_{2}$ state. We calculate the neutrino emissivity in each layer of stars.

We adopt the EoS which includes the first order phase transition between the hadronic phase and the quark phase[3]. We assume the dimensional structure of mixed phase at the transition. The maximum mass with this EoS is $2.13 M_{\odot}$ which satisfies recent $2 M_{\odot}$ observations [4, 5], even including the quark phase. The $M-R$ relation is shown in Figure 2. In the mixed phase, the dimensional structure is determined by Wigner-Seitz approximation, which fixes the cell size and the symetricity. The volume fraction of each phase can be calculated by the cell size, and it can determine the bulk neutrino emissivity. Also, the centre of the maximum mass star is constructed by mixed phase, it causes the neutrino emission processes of both of hadronic phase and quark phase are activated. In the hadronic phase, the proton fraction can exceed $1 / 9$ at high density region, the strong neutrino emission by the DU is activated. 
We choose three masses of stars as calculation models $\left(1.41,2.04\right.$, and $\left.2.13 M_{\odot}\right)$. And four kinds of neutron ${ }^{3} \mathrm{P}_{2}$ superfluid models and quark CSC paring (CFL, 2SC, and 2SC+X) as parameters. First, we set the surface composition as ${ }^{56} \mathrm{Fe}$, and calculate the cooling curves. And then we show the effect of light element surface composition for one fixed superfluid model.

\section{Results and discussions}
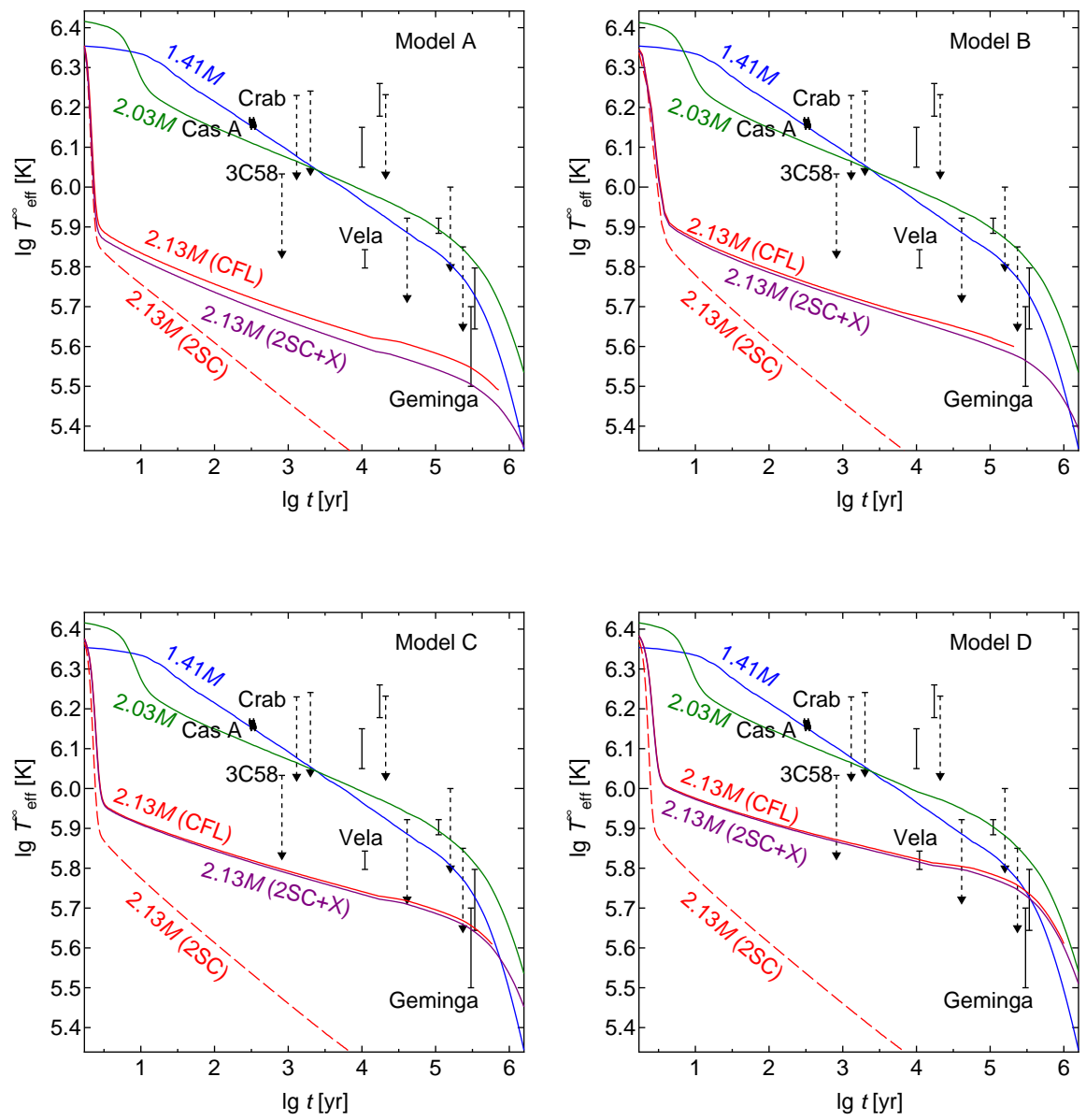

Figure 3. Cooling curves with various superfluid models. Blue (green) line denotes $1.41 M_{\odot}\left(2.03 M_{\odot}\right)$, respectively. Red solid (dashed) line denotes $2.13 M_{\odot}$ with CSC paring of CFL (2SC), respectively. Purple line denotes $2.13 M_{\odot}$ with $2 \mathrm{SC}+\mathrm{X}$ model. Each superfluid models are shown on the right-top corners of each panels.

We show the cooling curves of neutron stars with superfluid model A, B, C, and D with ${ }^{56} \mathrm{Fe}$ surface in Figure 3, and the curves with ${ }^{4} \mathrm{He}$ surface in Figure 4. The curves of $2.13 M_{\odot}$ show the difference of CSC: The red solid curves for CFL, the red dashed curves for $2 \mathrm{SC}$, and 
the purple solid curves for $2 \mathrm{SC}+\mathrm{X}$. In each models, heavy stars cool faster than lighter stars in early stage $\left(t<10^{3} \mathrm{yr}\right)$, and the medium mass star stays warm in late stage $\left(t>10^{3} \mathrm{yr}\right)$. Also, except the $2 \mathrm{SC}$ cases, the curves of $2.13 M_{\odot}$ are sensitive to the superfluid model of neutron ${ }^{3} P_{2}$ and they can explain cold neutron stars. The CSC paring affects to the cooling curves for the heaviest star model. With 2SC paring, the cooling curves locate low temperature region and they cannot match with observational data with the lower limit. With $2 \mathrm{SC}+\mathrm{X}$, the cooling curves are similar behaviour with CFL cases. Focusing on the observational data of Vela, the cooling curves of CFL and $2 \mathrm{SC}+\mathrm{X}$ can cross the data, but $2 \mathrm{SC}$ model cannot reach the lower limit value.

The results show that the CSC model has an impact on the cooling of neutron stars. If the CSC state realized in neutron stars is the 2SC state, heavy stars with quarks show very fast cooling, which cannot explain the currently observed isolated neutron stars. In contrast, considering CFL state, the neutrino emission by quarks is strongly suppressed, and the DU process of hadronic phase becomes dominant. The cooling curves of CFL state, can pass the low temperature observations in the cooling diagram. In the case of CFL and $2 \mathrm{SC}+\mathrm{X}$, the superfluidity of neutrons ${ }^{3} P_{2}$ has a significant influence on the cooling of neutron stars.

Considering CSC, there is one problem. The CFL paring suits for the cooling results which shows moderate strong cooling, but the appearing density is thought to be much higher density. On the other hand, the 2SC paring suits for the appearing density, but the cooling result shows too strong cooling for explaining the observation. The $2 \mathrm{SC}+\mathrm{X}$ phase can solve this problem. The appearing density of $2 \mathrm{SC}+\mathrm{X}$ suits for the density of neutron star, and it suppresses strong neutrino emission by quarks in all colours.

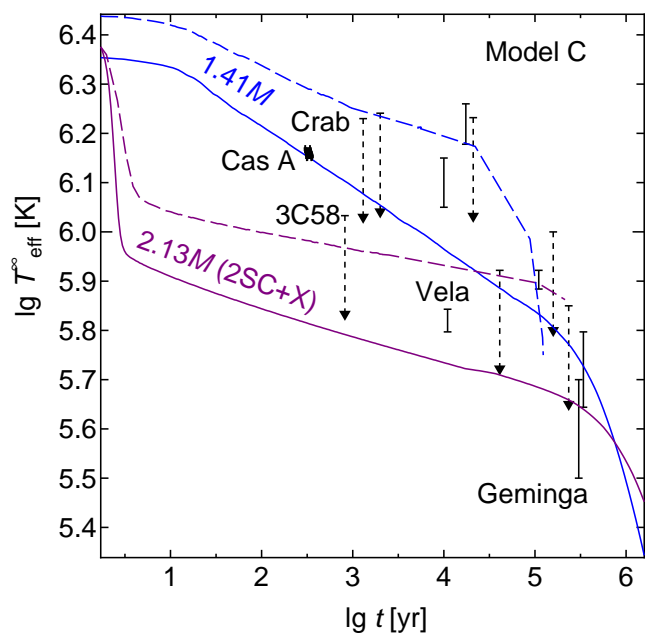

Figure 4. Cooling curves of $1.41 M_{\odot}$ and $2.13 M_{\odot}(2 \mathrm{SC}+\mathrm{X})$ with the light element surface, and with the Model C. Solid (dashed) curves denote ${ }^{56} \mathrm{Fe}\left({ }^{4} \mathrm{He}\right)$ surface composition, respectively.

\section{Summary}

We have demonstrated the neutron star cooling with quark hadron continuity. Considering the continuity, the $2 \mathrm{SC}+\mathrm{X}$ state can explain the cooling of neutron stars. Also we notice that the neutron ${ }^{3} P_{2}$ superfluidity which is continued by the $d$-quarks affects the thermal history of neutron star cooling. This trend does not change even if the surface composition is modified. 
We have calculated the cooling curves with light surface elements with the same model of the model $\mathrm{C}$ shown in Figure 4.

By the results of our calculation, the cold neutron star can be explained by CSC quark matter which causes strong neutrino emission. The neutrino emissivity is strong enough to drop the star temperature, but the appearance of CSC phase suppresses the emission rate and the cooling curves can pass the observed values. To consider neutron star cooling with quark phase, it is important to consider three "superstates" of neutrons, protons, and quarks. Also, the exotic states which we have not included, such as hyperon mixing or meson condensation, are possible candidates of strong cooling of neutron stars. Considering these states also require superfluid effect of corresponding particles. The study of the exotic states which realized in neutron stars should be discussed in the both of nuclear physics and astrophysics continuously.

\section{References}

[1] D. Page et al., Astrophys. J. Suppl. 155, 623 (2004).

[2] Y. Fujimoto et al., Phys. Rev. D. 101, 094009 (2020).

[3] N. Yasutake et al., J. Phys. Conf. Ser. 665, 012068 (2016).

[4] P. B. Demorest et al., Nature 467, 1081 (2010).

[5] J. Antoniadis et al., Science 340, 448 (2013). 